\title{
Hazard ratio of progression-free survival is an excellent predictor of overall survival in phase III randomized controlled trials evaluating the first-line chemotherapy for extensive-disease small-cell lung cancer
}

\author{
Hao Chen ${ }^{1}$, Nobuyuki Horita ${ }^{1}$, Kentaro Ito $^{2}$, Yu Hara ${ }^{1}$, Nobuaki Kobayashi ${ }^{1}$, Takeshi Kaneko ${ }^{1}$ \\ ${ }^{1}$ Department of Pulmonology, Yokohama City University Graduate School of Medicine, Yokohama, Japan; ${ }^{2}$ Respiratory Center, Matsusaka Municipal \\ Hospital, Matsusaka, Japan \\ Contributions: (I) Conception and design: N Horita, K Ito; (II) Administrative support: Y Hara, N Kobayashi, T Kaneko; (III) Provision of study \\ materials or patients: H Chen, N Horita; (IV) Collection and assembly of data: H Chen, N Horita; (V) Data analysis and interpretation: N Horita, K \\ Ito; (VI) Manuscript writing: All authors; (VII) Final approval of manuscript: All authors. \\ Correspondence to: Nobuyuki Horita, MD, PhD. Department of Pulmonology, Yokohama City University Graduate School of Medicine, 3-9 Fukuura, \\ Kanazawa-ku, Yokohama, 236-0004, Japan. Email: horitano@yokohama-cu.ac.jp.
}

\begin{abstract}
Background: Whether hazard ratio (HR) of progression-free survival (HRpfs), odds ratio (OR) of response rate (ORrr), OR of disease control rate (ORdcr), and OR of 1-year overall survival (ORos1y) used for extensive-disease small-cell lung cancer (ED-SCLC) correlate with HR of overall survival (HRos) at a randomized-trial level, especially for a trial that evaluates molecular-targeted therapy (MTT) or immunecheckpoint inhibitor (ICI), is unclear.

Methods: We included an individually randomized controlled trial (RCT) comparing two regimens as the first-line treatment for chemo-naive ED-SCLC, which have been reported in English-language since 2000. A weighted Spearman's rank correlation coefficient (r) was evaluated.

Results: We finally found 42 eligible articles consisted of 11,478 cases. Estimated $r$ with HRos were as followings: HRpfs (29 trial, 8,573 cases, r=0.87), ORrr (39 trials, 11,030 cases, r=0.47), ORdcr (29 trials, 7,799 cases, $r=0.48$ ), and ORos1y (40 trials, 11,250 cases, $r=0.69$ ). Phase III subgroup (16 trials, 7,079 cases) yielded an excellent $r$ between HRpfs and HRos of 0.96. ORder presented the best correlation with HRos for phase II trial subgroup ( $r=-0.64$ ); however, this result was mainly calculated from MTT trials. HRpfs may overestimate the efficacy of MMT in a phase II trial. ORrr and ORder might undervalue the efficacy of ICI even in a phase III trial.

Conclusions: HRpfs can be a good surrogate of HRos, especially in a phase III trial. Depending on a single outcome in a randomized phase II trial may result in unneeded phase III trial or inappropriate abandonment of the regimen.
\end{abstract}

Keywords: Small-cell lung carcinoma; survival; treatment outcome; molecular targeted therapy

Submitted Mar 02, 2020. Accepted for publication Jun 29, 2020.

doi: $10.21037 /$ tlcr-20-377

View this article at: http://dx.doi.org/10.21037/tlcr-20-377

\section{Introduction}

Small-cell lung cancer (SCLC) is a malignant respiratory disease usually preceded by smoking habit (1). Most patients were given the initial diagnosis of extensive-disease (ED)-
SCLC because it grows and disseminates before a patient recognizes symptoms such as cough, sputum, and dyspnea. A patient with ED-SCLC is usually treated with systemic chemotherapy as SCLC is a chemotherapy-amenable 
malignancy. However, ED-SCLC is not a curable disease. Therefore, the goal of treatment is to prolong survival; thus, overall survival (OS) is the standard outcome to evaluate a chemotherapy regimen.

Although OS is the most widely accepted endpoint for a randomized trial examining the efficacy of chemotherapy for lung cancer and hazard ratio (HR) is the most robust statistic to assess the time to event outcome in a randomized trial (2), some investigators prefer progression-free survival (PFS), response rate (RR), disease control rate (DCR), and milestone 1-year OS (OS1y) instead of HR of OS (HRos) because calculating HRos requires a long-term followup $(3,4)$. Whether these surrogate endpoints accurately reflect HRos in an RCT assessing the chemotherapy for ED-SCLC is a serious concern because using an unreliable surrogate endpoint in an RCT critically diminishes the trustworthiness of the result. The validity of these surrogate endpoints was frequently evaluated at an individual level (5-10). However, it has not been sufficiently evaluated at a trial level. In addition, it is still not clear if these surrogate endpoints are useful for a trial that evaluates moleculartargeted therapy (MTT) and immune-checkpoint inhibitor (ICI), which have been featured recently. The goal of the current research is to examine how HR of PFS (HRpfs), odds ratio (OR) of RR (ORrr), OR of DCR (ORdcr), and OR of OS1y (ORos1y) correlate with HRos at a randomized-trial level. The authors present the following article in accordance with the PRISMA reporting checklist (available at http://dx.doi.org/10.21037/tlcr-20-377).

\section{Methods}

\section{Protocol registration}

This protocol of the systematic review has been submitted to the website of International Prospective Register of Systematic Review (ID: 154051) (11). We have composited this protocol following the Preferred Reporting Items for Systematic Reviews and Meta-Analyses statement (12).

\section{Study search}

Search formulas for PubMed, Web of Science Core Collection, Cochrane, and EMBASE are presented as Supplementary Text 1 . The search was done on October 10th, 2019. An additional manual search was conducted by two investigators ( $\mathrm{HC}$ and $\mathrm{NH}$ ) independently.

Candidate articles were first screened and then scrutinized independently by two investigators.
A trial that included both limited disease and ED was included as long as the data for ED cases were separately extractable.

\section{Inclusion criteria, publication type and trial design}

We included an individually randomized controlled trial (RCT) comparing two regimens as the first-line treatment for chemo-naive ED-SCLC, which have been reported and published in English-language full papers since 2000. Reports before 2000 were not interest to us because chemotherapy regimens and imaging modalities before 2000 are clearly outdated. English-language conference abstract published after 2015 was also acceptable to collect data for MTT and ICI trials.

Included patients should be randomized before the chemotherapy initiation. Therefore, randomization after a few cycles of chemotherapy was not allowed.

A trial assessing a specific population defined by age, race, and performance status was permitted.

\section{Inclusion criteria, treatments}

A regimen that consisted of cytotoxic agent, MTT, ICI, and combination of these drugs was allowed. However, any regimen that included cytotoxic reagents developed around 1950, so-called the first-generation anticancer drugs, namely Methotrexate, Mitomycin, Vincristine, Cyclophosphamide, Doxorubicin, and Ifosfamide were excluded from our analysis because such regimens are outdated.

A three-arm trial was not included because we should not arbitrarily select two arms form the three arms.

\section{Inclusion criteria, patients}

Chemo-naive patients with ED-SCLC who underwent first-line chemotherapy were included.

\section{Quality assessment}

The quality of an original study was scored using six domains of the Cochrane Risk of Bias: random sequence generation, allocation concealment, performance, detection, attrition, reporting (13).

\section{Outcomes}

How HRpfs, ORrr, ORder, and ORos1y correlated with 
HRos was evaluated. Then, subgroup analysis based on study phase was conducted.

Trials were further subdivided into three subgroups according to treatment regimens. A "MTT trial", which compared platinum doublet plus MTT versus doublet alone, belonged to "MTT subgroup". "The ICI subgroup" consisted of "ICI trials" focusing on adding an ICI on platinum doublet. No study directly compared two MTT regimens, two ICI regimens, or an MTT regimen versus an ICI regimen. A "cytotoxic-drug trial", which compared two cytotoxic regimens without MTT and ICI, consisted "cytotoxic drug subgroup".

\section{Data extraction}

Data for included studies, such as author name, publication year, country of origin, numbers of patients randomized, chemotherapy regimen, and outcomes were extracted by the two investigators ( $\mathrm{HC}$ and $\mathrm{NH}$ ) independently. The data extracted by the two investigators were cross-checked and any discrepancies were discussed between them. When necessary, we adopted Parmar's method to extract data from Kaplan-Meier curves (14). Intention-to-treat analysis was preferred over full-analysis-set analysis and per-protocol analysis when two or more of them were available. An updated survival data might be used.

\section{Statistical analyses}

RR and DCR were calculate in the standard manner (15). A weighted Spearman's rank correlation coefficient (r) between outcomes was calculated using "cor_spem" command in the "boot" package of R (16) (R foundation, Vienna, Austria). Correlation coefficient, $\mathrm{r}$, which ranges -1 to 1 is usually considered as followings: $|\mathrm{r}|<0.2$, meaningless correlation; $0.2<|\mathrm{r}|<0.4$, week correlation; $0.4<|\mathrm{r}|<0.6$, moderate correlation; $0.6<|\mathrm{r}|<0.8$, strong correlation; $0.8<|\mathrm{r}|<0.9$, very strong correlation; $0.9<|\mathrm{r}|$, excellent correlation.

A weighted regression line and a determination coefficient, $\mathrm{R}^{2}$, were calculated with the " $\mathrm{lm}$ " command of $\mathrm{R}$ software after logarithmization (17).

\section{Results}

\section{Study selection and characteristics}

We first found 1,431 articles from database searches and 5 articles from hand searches, respectively. Of 1,436 articles that met the preliminary criteria, 569, 669, and 156 were excluded through removal of duplication, title/abstract screening, and whole article scrutinizing, respectively (Figure 1). Author group of the CASPIN trial provided detailed unpublished data (18). We finally found 42 eligible articles, of which 29, 39, 29, and 40 provided data for HRpfs, ORrr, ORdcr, ORos1y (Table 1, Supplementary Text 2). The total number of ED-SCLC cases across all trial was 11,478 .

Trials were reported from USA $(\mathrm{N}=16), \mathrm{EU}(\mathrm{N}=13$ including 2 reports from $\mathrm{UK})$, Japan $(\mathrm{N}=6)$, China $(\mathrm{N}=4)$, Korea $(\mathrm{N}=2)$, and India $(\mathrm{N}=1)$.

\section{HR of PFS}

Weighted Spearman's rank correlation, r, yielded from 29 trials with 8,573 ED-SCLC cases was 0.87 , which suggests a very strong correlation between HRpfs and HRos (Figure $2 \mathrm{~A}$ ). The following regression formula was provided, $\log ($ HRos $)=\log ($ HRpfs $) \times 0.683-0.013$ as shown in Figure $2 A$. Coefficient of determination, $\mathrm{R}^{2}$ was 0.72 , suggesting that HRpfs could explain $72 \%$ of HRos outcome.

Phase III subgroup (16 trials, 7,079 cases) yielded $r$ of 0.96 and $\mathrm{R}^{2}$ of 0.90 , which meant excellent correlation between HRpfs and HRos (Figure 2B). Sixteen phase III RCTs consisted of 11 cytotoxic-drug trials, two MTT trials, and three ICI trials (Figure 2B).

Phase II subgroup included three cytotoxic-drug trials, eight MTT trials and no ICI trial (Figure 2C). The correlation coefficient yielded from these 11 phase II trials was week $(\mathrm{r}=0.26)$ (Figure 2C) partly because MTT trials were widely scattered left upper area, wherein HRos is not as good as expected from HRpfs.

\section{Odds ratio of response rate and disease control rate}

$\operatorname{ORrr}(\mathrm{N}=39, \mathrm{n}=11,030, \mathrm{r}=-0.47$, Figure $2 D, E, F)$ and ORder $(\mathrm{N}=29, \mathrm{n}=7,799, \mathrm{r}=-0.48$, Figure $2 G, H, I)$ had moderate correlation with HRos. Phase-based subgroup analyses did not reveal considerable difference between $r$ between phase III and phase II subgroups (Figure 2E,F,H,I). ORdcr consistently showed higher $|\mathrm{r}|$ than ORrr in all-trial, phase II, and phase III analyses (Figure 2G,H,I).

ORrr and ORdcr data regarding ICI were obtainable from three and two studies, respectively, all of which were phase III trials (Figure 2E,H). Based on these limited trials, ORrr and ORdcr did not seem to reflect HRos. Of note, 
PRISMA 2009 Flow Diagram
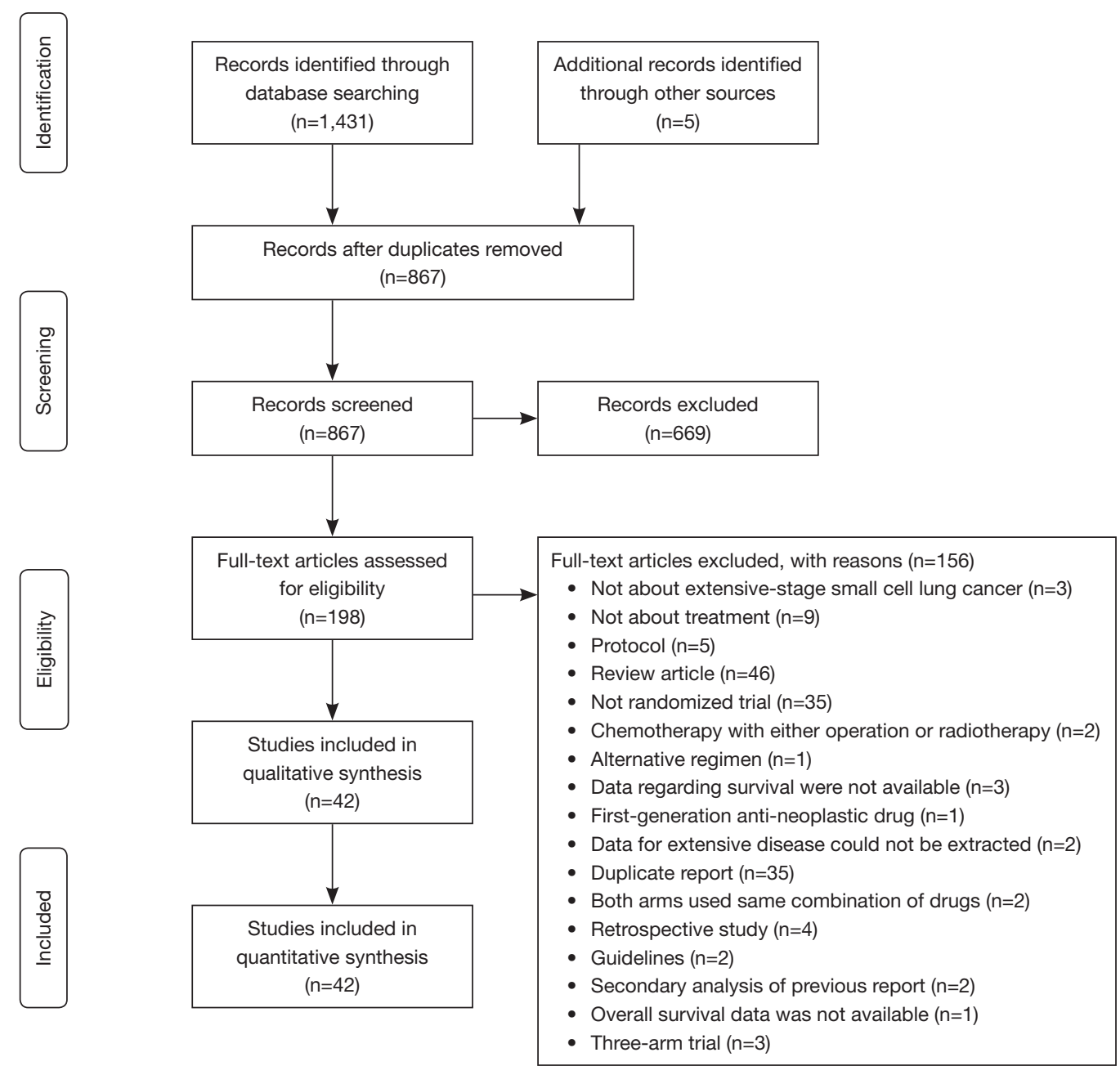

Figure 1 PRISMA flow chart for study selection.

PD-L1 trials were located considerably below the regression line in the ORrr-HRos plot and ORder-HRos plot (Figure 2E,H). This meant PD-L1 regimens led to longer OS than expected from response or disease-control evaluation. That is, RR and DCR evaluation might underestimate the efficacy of PD-L1 regimen.

\section{Odds ratio of 1-year OS}

Forty RCTs with 11,250 cases yielded $\mathrm{r}$ of -0.69 between
ORos1y and HRos, meaning a strong correlation (Figure 27). The coefficien $\mathrm{r}$ were -0.76 in phase III subgroup (Figure $2 K$ ) and -0.42 in phaseII subgroup (Figure 2L).

\section{Discussion}

We gathered the outcome data from 42 two-arm randomized trials consisted of 11,478 patients with EDSCLC and examined how HRpfs, ORrr, ORder, and 


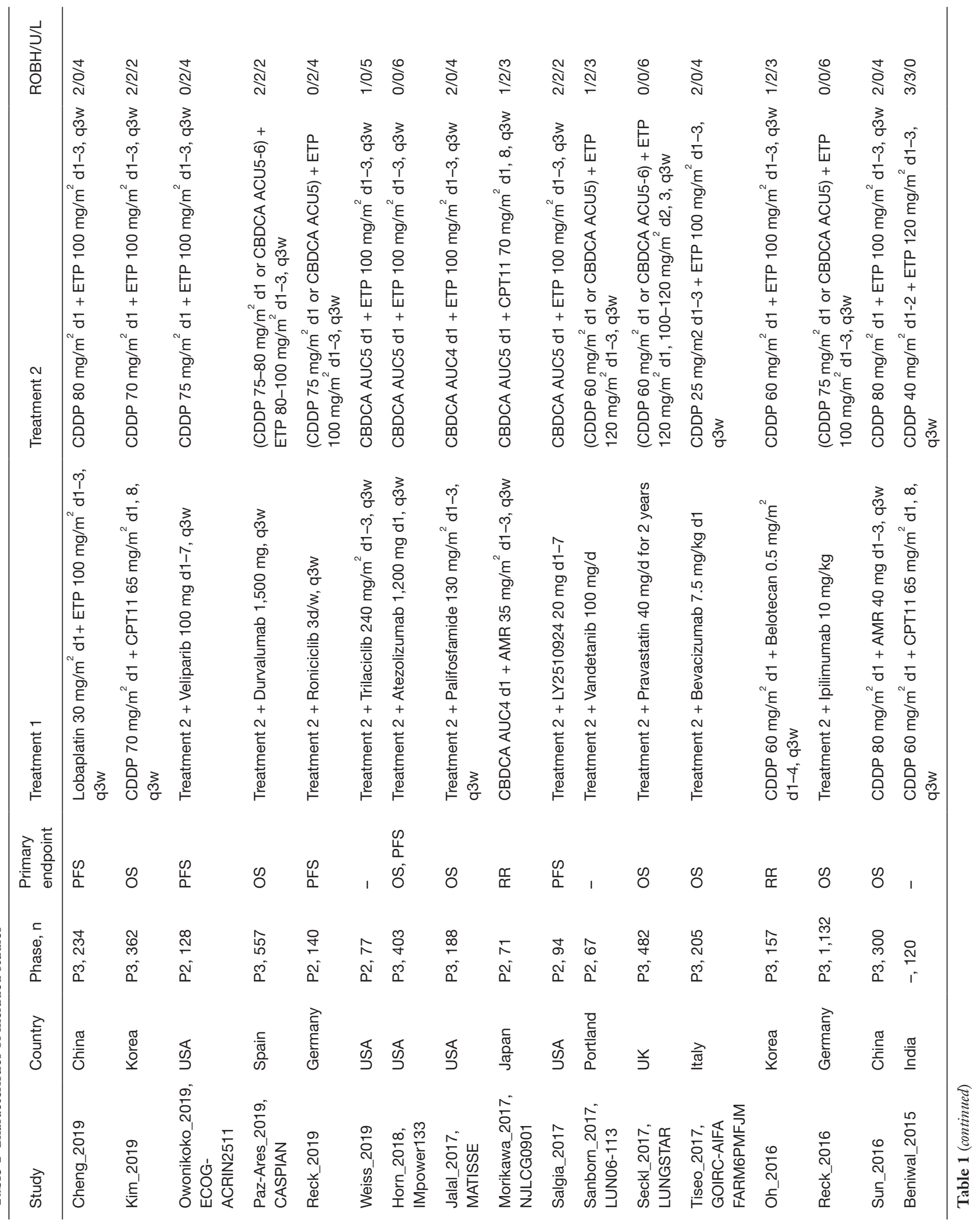




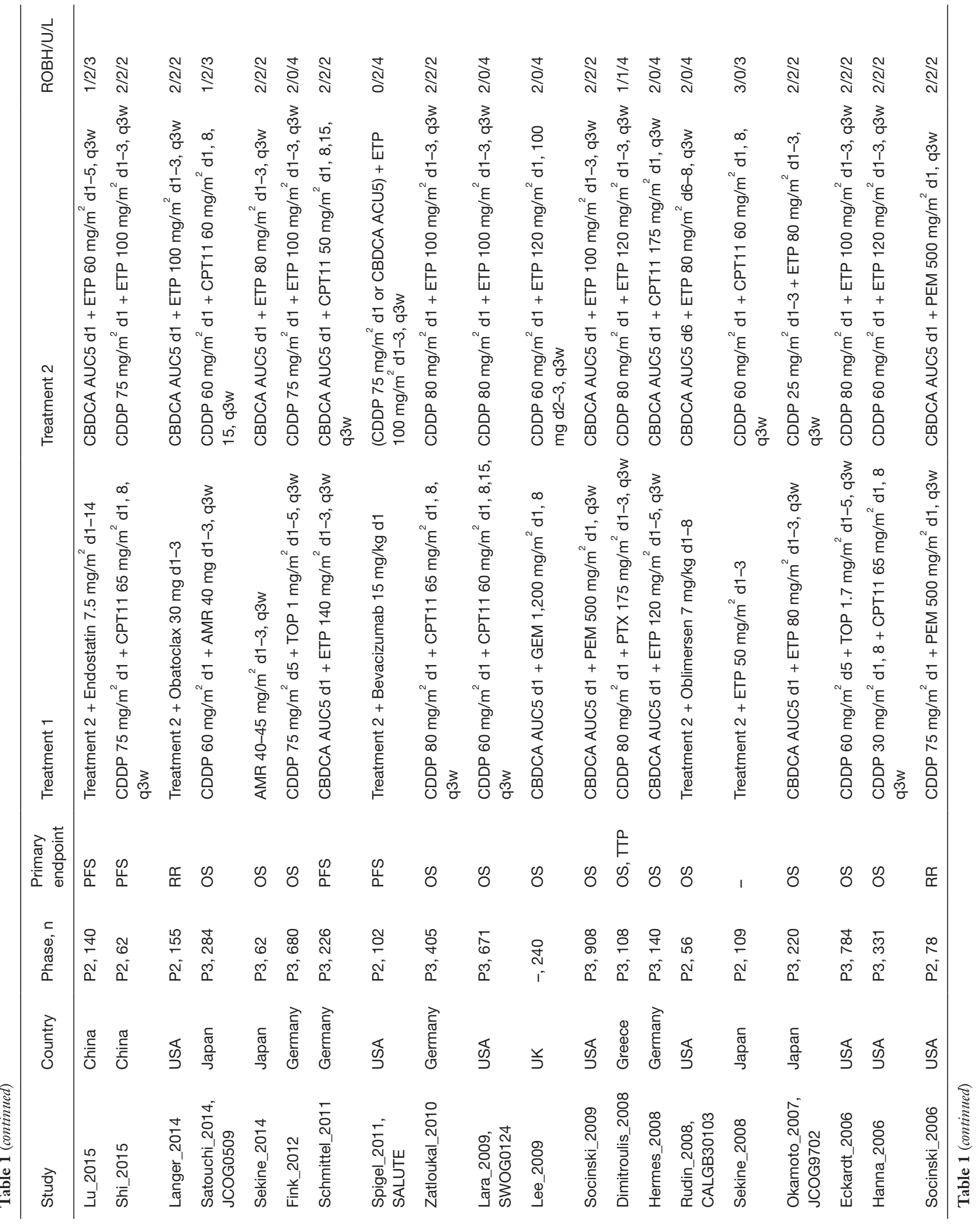




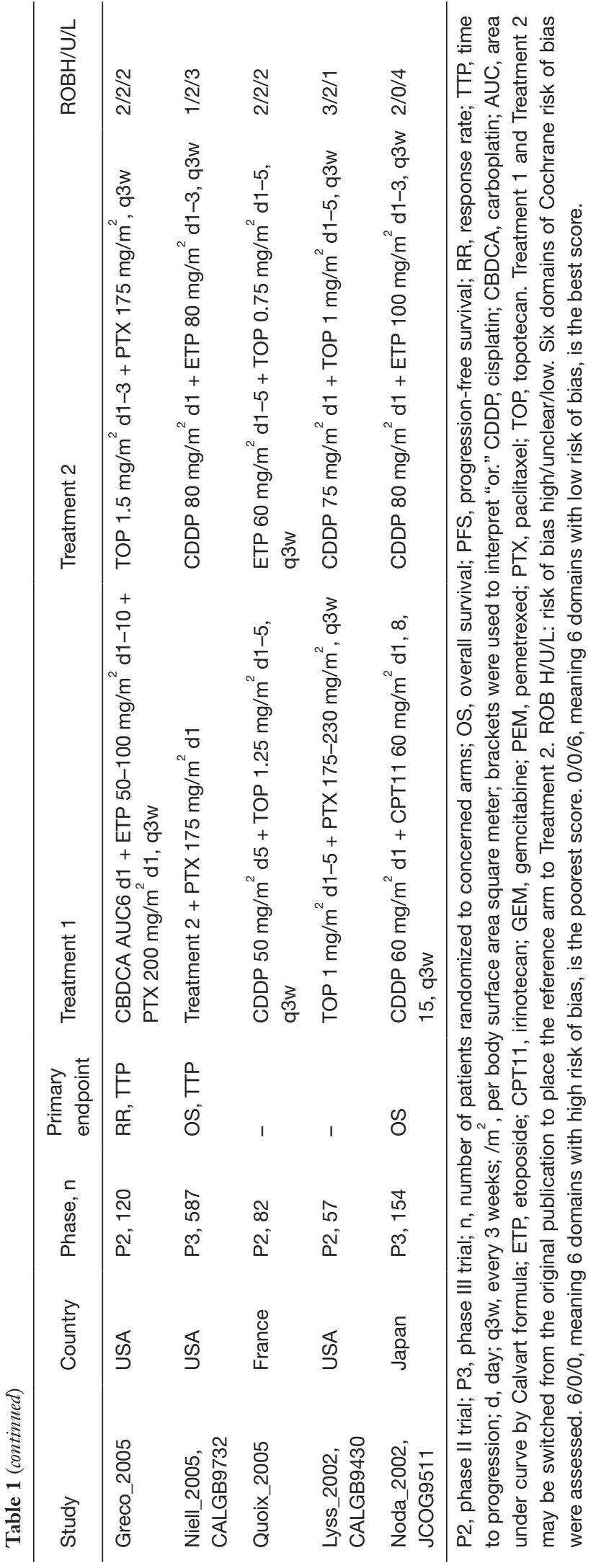

ORos1y reflected HRos. RR and DCR are easily available in two months of patient entry. However, they had only moderate correlation with HRos. Milestone OS1y is often featured in non-small cell lung cancer trials as a surrogacy of long-term survival, especially ICI-treated patients $(3,4)$. However, late-phase OS plateau has not been observed in a cohort of ICI-treated ED-SCLC cases $(18,19)$, thus OS1y does not presumed a long survival of ED-SCLC cases. Besides, OS1y is not a robust statistic because OS1y represents OS data at a single time point though OS1y is a biomarker that is directly derived from OS survival curve. One year after the entry of the last patient, OS and PFS curves may be sufficiently mature. Thus, we need not use milestone OS1y for the first-line ED-SCLC trial. In contrast, HRpfs, which had very strong correlation with HRos ( $\mathrm{r}=0.89$ ), is a reasonable surrogacy of HRos.

In the phase III setting, $r$ between HRpfs and HRos was as high as 0.96 and $R^{2}$ between them was 0.90 . In other words, PFS alone almost determined OS in a phase III trial for the 1st-line ED-SCLC. This is comparable with the fact that sensitivity to the first-line chemotherapy predict the response to later-line chemotherapy and post-progression survival (20). This excellent $r$ was also supported large number of randomized patients in a phase III trial. As OS of an ED-SCLC case has recently been becoming longer thanks to ICI, evaluating HRos demands extended followup $(18,19)$. HRpfs can be a desirable surrogate outcome in a future phase III trial.

In a randomized phase II trial, PFS and RR were often selected as the primary outcome. Nonetheless, HRpfs did not correlate well with HRos in phase II trials (Figure 2C). Furthermore, we do not have sufficient data to clarify how HRpfs is useful for phase II ICI trial and cytotoxic-drug trial because most of randomized phase-II trials were MTT trials (Figure 2C). ORder showed higher $|\mathrm{r}|$ of 0.64 with HRos in the phase II setting compared to that of ORrr $(|\mathrm{r}|=0.41)$. Lara $e t$ al. showed that DCR is a better patientlevel surrogate of OS compared to RR in the ED-SCLC in the second-line setting (5). They also analyzed SCLC cases who underwent the first-line platinum doublet and found that DCR better predicts OS than RR does because patients who had DCR had similar OS with those who had a response. RR is usually a more preferred outcome than DCR (Table 1); however, DCR may be another reasonable option. In any case, none of RR, DCR, and PFS cannot warrant OS outcome in the phase II setting. Relying on a single outcome in the randomized phase II trials to start or to dismiss phase III trial might be risky. 
A

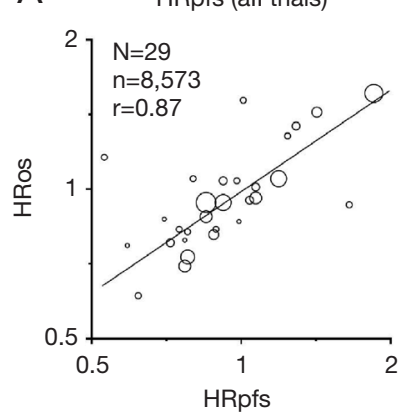

D

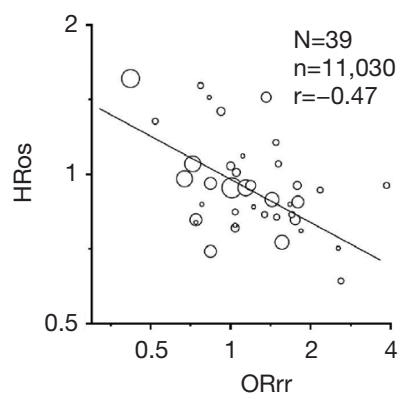

G
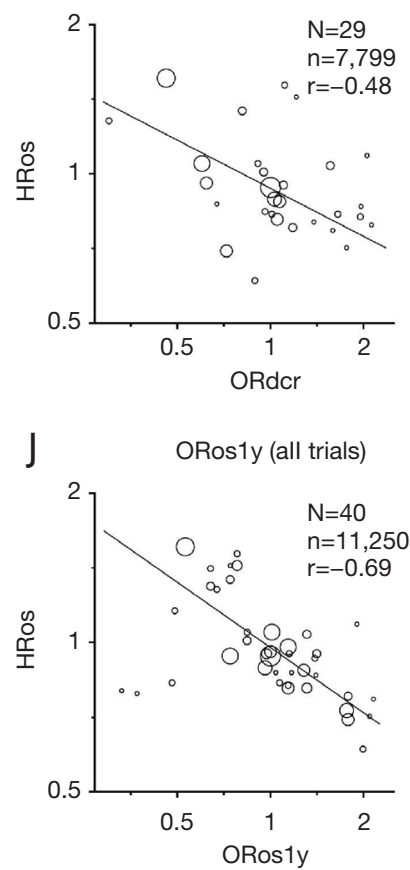

B

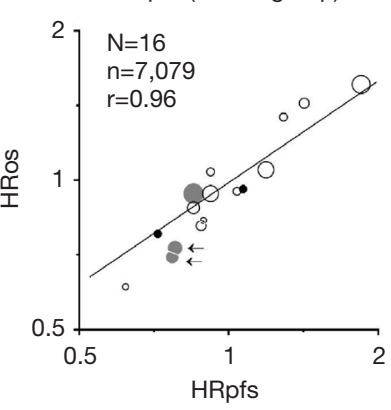

E

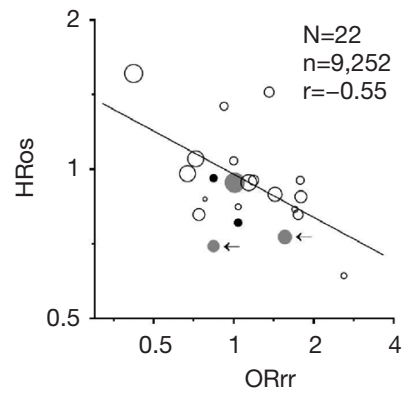

H ORdcr (P3 subgroup)

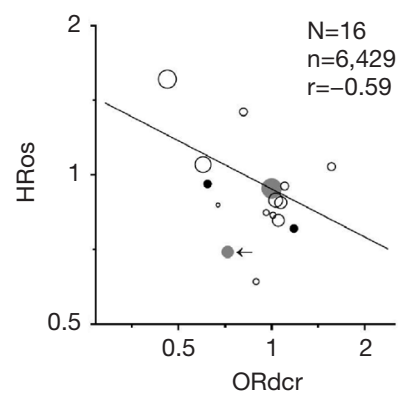

K ORos1y (P3 subgroup)

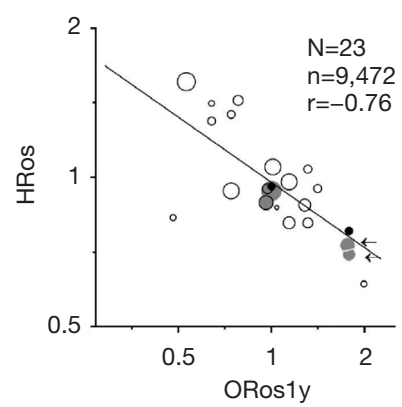

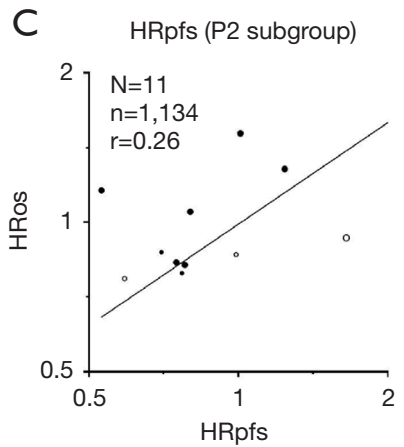

F ORrr (P2 subgroup)

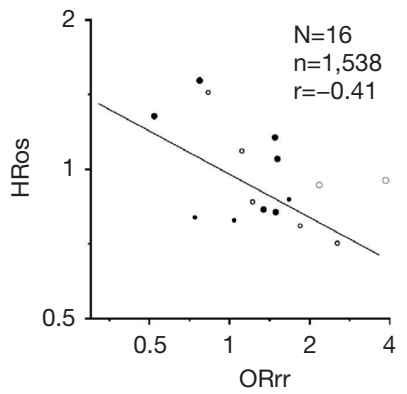

I

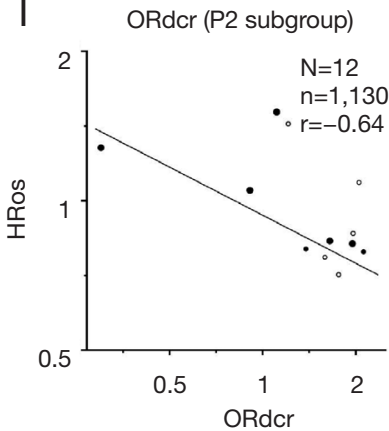

$\mathrm{L}$

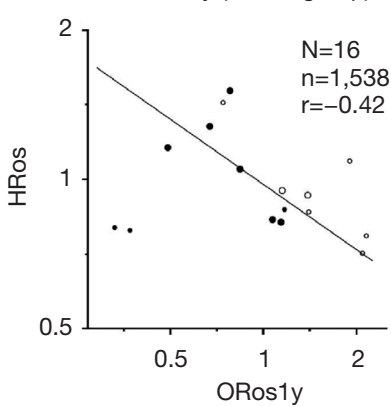

Figure 2 Correlation between surrogate outcomes and hazard ratio of overall survival. Each circle represents a randomized trial and a size of the circle represents a sample size. A line in the scatter plot is a regression line after logarithmization based on the all trials as shown in the left panel $(A, D, G, J)$. The same regression line is drawn for the other panel. In a scatter plot of P3 and P2 subgroups (middle and right panels), an open circle indicates a cytotoxic-drug trial, a filled circle indicates molecular-targeted therapy, and a grey circle indicates an immune checkpoint inhibitor trial, and left pointing arrow indicates PD-L1 trial. N, number of trials; n, number of patients in a trial; r, Weighted Spearman's rank correlation coefficient; HRos, hazard ratio of overall survival; HRfps, hazard ratio of progression-free survival; ORrr, odds ratio of response rate; ORdcr, odds ratio of disease control ratio; P3, phase III; P2, phase II. 
Although not conclusive because of a limited number of ICI trials, neither ORrr nor ORder could not predict HRos in ICI trials. Of note, ORrr and ORder in PD-L1 trials underestimated HRos of PD-L1 trials (Figure 2F,I). Surprisingly, patients treated by atezolizumab regimen in IMpower133 trial had longer survival with HRos of 0.70 and HRpfs of 0.77 though RR and DCR in atezolizumab arm was lower than those in the standard arm (19). In the era of ICI, RR and DCR might fall into desuetude.

We need to discuss agreement and disagreement between our data and published data. In 2009, Hotta et al. revealed that RR difference was modestly associated with the mean survival time difference $\left(\mathrm{R}^{2}=0.3314\right)$ at trial-level using data from 48 phase III trials (7). $R^{2}=0.3314$ is roughly equivalent to correlation coefficient of 0.58 , which does not conflict with $\mathrm{r}=-0.47$ between ORrr and HRos in our analysis (Figure 2D).

A report by Foster et al. in 2011 showed that progressionfree at 4 and 6 months was associated with OS at the individual level. This supports that HRpfs reflect OS as shown in our analysis (Figure 2A) (8). They also divided data of three trials into 32 unit-level components and described that HRpfs and HRos had $\mathrm{r}$ of 0.73 , which does not conflict with $r$ of 0.87 between HRpfs and HRos in our analysis. In 2015 , Foster et al. also reported that weighted least square $\mathrm{R}^{2}$ was 0.83 between HRpfs and HRos using the data of 7 trials (10), which was compatible with $\mathrm{R}^{2}=0.72$ between HRpfs and HRos in our analysis(Figure $2 A$ ). Because our data were largely enriched by a larger number of trials including MTT trials and ICI trials, our analysis provides useful information for future trial designing.

Imai $e t a l$. reported that PFS was moderately correlated with OS of 0.58 at the patient level (9). Some may think that these coefficients may seem poorer than our data $(\mathrm{r}=0.87$, Figure 2A). However, patient level OS and PFS are clearly less robust and strong correlation is rarely achieved. Thus, the discrepancy between Imai and us is explainable.

In 2014, Nickolich analyzed 66 trials that were published until 2010 and did not find significant correlation between PFS and OS of ED-SCLC cases at trial level (unweighted Pearson's correlation coefficient $=0.369)$ (6). The coefficient estimated by Nickolich may seem much lower than that in our analysis $(\mathrm{r}=0.87$, Figure $2 A)$. This large discrepancy may be introduced by methodology difference. We believe that the weighted Spearman's rank correlation coefficient, which we applied, is a reasonable and robust statistic to evaluate the correlation using data of trials with a variety of sample sizes.

One limitation of our study is that surrogacy of outcomes in a ICI trial could not be sufficiently evaluated because of the limited number of trials. Another is that the result concerning phase II RCT was largely driven by MTT trials.

In conclusion, when all trials were analyzed collectively, HRpfs very strongly correlate with HRos $\left(\mathrm{r}=0.87, \mathrm{R}^{2}=0.72\right.$, Figure $2 A$ ) at the randomized trial level. In a phase III subgroup, the correlation was excellent $\left(r=0.96, R^{2}=0.90\right.$, Figure 2B). HRpfs is an excellent surrogate outcome of HRos, especially in a phase III trial. ORder presented the best correlation with HRos for randomized phase II trials (Figure 2I, $\mathrm{r}=-0.64$ ). However, this correlation did not reach the level of very strong correlation. Besides, this result was mainly calculated from MTT trials (Figure 2I). Depending on a single outcome in a randomized phase II trial may result in unneeded phase III trial or inappropriate abandonment of the regimen. For a phase III ICI trial, PFS seems a reasonable surrogate of OS (Figure $2 B$ ), but RR (Figure 2E) and DCR (Figure 2H) undervalue OS. PFS often overestimate the efficacy of MTT in a randomized phase II trial (Figure 2C).

\section{Acknowledgments}

Funding: None.

\section{Footnote:}

Reporting Checklist: The authors have completed the PRISMA reporting checklist. Available at http://dx.doi. org/10.21037/tlcr-20-377

Conflicts of Interest: All authors have completed the ICMJE uniform disclosure form (available at http://dx.doi. org/10.21037/tlcr-20-377). The authors have no conflicts of interest to declare.

Ethical Statement: The authors are accountable for all aspects of the work in ensuring that questions related to the accuracy or integrity of any part of the work are appropriately investigated and resolved.

Open Access Statement: This is an Open Access article distributed in accordance with the Creative Commons Attribution-NonCommercial-NoDerivs 4.0 International License (CC BY-NC-ND 4.0), which permits the noncommercial replication and distribution of the article with the strict proviso that no changes or edits are made and the original work is properly cited (including links to both the 
formal publication through the relevant DOI and the license). See: https://creativecommons.org/licenses/by-nc-nd/4.0/.

\section{References}

1. Jackman DM, Johnson BE. Small-cell lung cancer. Lancet 2005;366:1385-96.

2. Nakashima K, Horita N, Nagai K, et al. ProgressionFree Survival, Response Rate, and Disease Control Rate as Predictors of Overall Survival in Phase III Randomized Controlled Trials Evaluating the First-Line Chemotherapy for Advanced, Locally Advanced, and Recurrent Non-Small Cell Lung Carcinoma. J Thorac Oncol 2016;11:1574-85.

3. Zhao S, Zhang Z, Zhang Y, et al. Progression-free survival and one-year milestone survival as surrogates for overall survival in previously treated advanced non-small cell lung cancer. Int J Cancer 2019;144:2854-66.

4. Blumenthal GM, Zhang L, Zhang H, et al. Milestone Analyses of Immune Checkpoint Inhibitors, Targeted Therapy, and Conventional Therapy in Metastatic NonSmall Cell Lung Cancer Trials: A Meta-analysis. JAMA Oncol 2017;3:e171029.

5. Lara PN Jr, Moon J, Redman MW, et al. Disease Control Rate at 8 Weeks Predicts Subsequent Survival in PlatinumTreated Extensive Stage Small-Cell Lung Cancer: Results From the Southwest Oncology Group (SWOG) Database. Clin Lung Cancer 2016;17:113-8.e1-2.

6. Nickolich M, Babakoohi S, Fu P, et al. Clinical trial design in small cell lung cancer: surrogate end points and statistical evolution. Clin Lung Cancer 2014;15:207-12.

7. Hotta K, Kiura K, Fujiwara Y, et al. Association between incremental gains in the objective response rate and survival improvement in phase III trials of first-line chemotherapy for extensive disease small-cell lung cancer. Ann Oncol 2009;20:829-34.

8. Foster NR, Qi Y, Shi Q, et al. Tumor response and progression-free survival as potential surrogate endpoints for overall survival in extensive stage small-cell lung cancer: findings on the basis of North Central Cancer Treatment Group trials. Cancer 2011;117:1262-71.

9. Imai H, Mori K, Wakuda K, et al. Progression-free survival, post-progression survival, and tumor response as surrogate markers for overall survival in patients with extensive small cell lung cancer. Ann Thorac Med 2015;10:61-6.

10. Foster NR, Renfro LA, Schild SE, et al. Multitrial Evaluation of Progression-Free Survival as a Surrogate
End Point for Overall Survival in First-Line ExtensiveStage Small-Cell Lung Cancer. J Thorac Oncol 2015;10:1099-106.

11. Booth A, Clarke M, Dooley G, et al. The nuts and bolts of PROSPERO: an international prospective register of systematic reviews. Syst Rev 2012;1:2.

12. Moher D, Liberati A, Tetzlaff J, Altman DG. Preferred reporting items for systematic reviews and meta-analyses: the PRISMA statement. BMJ 2009;339:b2535.

13. multicenter collaboration group of Department of Science and Technology of Guangdong Province and Health Commission of Guangdong Province for chloroquine in the treatment of novel coronavirus pneumonia. Expert consensus on chloroquine phosphate for the treatment of novel coronavirus pneumonia. Zhonghua Jie $\mathrm{He} \mathrm{He} \mathrm{Hu} \mathrm{Xi}$ Za Zhi 2020;43:E019.

14. Parmar MK, Torri V, Stewart L. Extracting summary statistics to perform meta-analyses of the published literature for survival endpoints. Stat Med 1998;17:2815-34.

15. Eisenhauer EA, Therasse P, Bogaerts J, et al. New response evaluation criteria in solid tumours: revised RECIST guideline (version 1.1). Eur J Cancer 2009;45:228-47.

16. Ripley B. Package 'boot'. February 23, 2016. Available online: https://cran.r-project.org/web/ packages/boot/boot.pdf\#search='corr+boot+r', accessed Dec 1, 2019.

17. $\mathrm{R}$ documentation: Fitting linear models. Available online: https://stat.ethz.ch/R-manual/R-devel/library/stats/html/ lm.html, accessed Dec 1, 2019.

18. Zhu N, Zhang D, Wang W, et al. A Novel Coronavirus from Patients with Pneumonia in China, 2019. The N Engl J Med 2020;382:727-33.

19. Horn L, Mansfield AS, Szczęsna A, et al. First-line atezolizumab plus chemotherapy in extensive-stage smallcell lung cancer. N Engl J Med 2018;379:2220-9.

20. Giaccone G, Donadio M, Bonardi G, et al. Teniposide in the treatment of small-cell lung cancer: the influence of prior chemotherapy. J Clin Oncol 1988;6:1264-70.

Cite this article as: Chen H, Horita N, Ito K, Hara Y, Kobayashi N, Kaneko T. Hazard ratio of progression-free survival is an excellent predictor of overall survival in phase III randomized controlled trials evaluating the first-line chemotherapy for extensive-disease small-cell lung cancer. Transl Lung Cancer Res 2020;9(4):1333-1342. doi: 10.21037/tlcr20-377 
Supplementary Text 1: Search formulas

PubMed

(Small cell lung cancer [title] OR small cell lung carcinoma [title] OR SCLC[title]) AND (ED OR extended OR extensive) AND (randomized OR randomised OR RCT OR phase 3 OR phase III OR randomly OR blinded OR blind OR placebo) and (Doxorubicin or Adriamycin or Epirubicine or Ifosfamide or Topotecan or Irinotecan or Etoposide or Cisplatin or Gemcitabin or Carboplatin or Teniposide or Epirubicin or Paclitaxel or apatinib or amrubicin or LY2510924 or Bevacizumab or Vismodegib or Cixutumumab or teniposide or Adriamycin or vindesine or nivolumab or ipilimumab or Atezolizumab OR Pembrolizumab OR Avelumab OR Durvalumab) not (NSCLC[title] or non-small[title])

Restricted by "publication since 2000" and "English language".

\section{Web of Science}

\#1 TI=(Small cell lung cancer OR small cell lung carcinoma OR SCLC) AND TS=(ED OR extended OR extensive) AND TS=(randomized OR randomised OR RCT OR phase 3 OR phase III OR randomly OR blinded OR blind OR placebo) and TS=(Doxorubicin or Adriamycin or Epirubicine or Ifosfamide or Topotecan or Irinotecan or Etoposide or Cisplatin or Gemcitabin or Carboplatin or Teniposide or Epirubicin or Paclitaxel or apatinib or amrubicin or LY2510924 or Bevacizumab or Vismodegib or Cixutumumab or teniposide or Adriamycin or vindesine or nivolumab or ipilimumab or Atezolizumab OR Pembrolizumab OR Avelumab OR Durvalumab) NOT TI $=($ NSCLC or non-small)

Restricted by "English", "Article" and "2000-2019".

\#2 TI=(Small cell lung cancer OR small cell lung carcinoma OR SCLC) AND TS $=($ ED OR extended OR extensive) AND TS=(randomized OR randomised OR RCT OR phase 3 OR phase III OR randomly OR blinded OR blind OR placebo) and TS=(Doxorubicin or Adriamycin or Epirubicine or Ifosfamide or Topotecan or Irinotecan or Etoposide or Cisplatin or Gemcitabin or Carboplatin or Teniposide or Epirubicin or Paclitaxel or apatinib or amrubicin or LY2510924 or Bevacizumab or Vismodegib or Cixutumumab or teniposide or Adriamycin or vindesine or nivolumab or ipilimumab or Atezolizumab OR Pembrolizumab OR Avelumab OR Durvalumab) NOT TI $=($ NSCLC or non-small)
Restricted by "English", "Letter/Meeting abstract" and "2015-2019."

\#3 \#1 or \#2

\section{Cochrane Search Manager}

\#1 Small cell lung cancer:ti OR small cell lung carcinoma:ti OR SCLC:ti

\#2 ED OR extended OR extensive

\#3 randomized OR randomised OR RCT OR phase 3 OR phase III OR randomly OR blinded OR blind OR placebo

\#4 Doxorubicin or adoriamicyn or Epirubicine or Ifosfamide or Topotecan or Irinotecan or Etoposide or Cisplatin or Gemcitabin or Carboplatin or Teniposide or Epirubicin or Paclitaxel or apatinib or amrubicin or LY2510924 or Bevacizumab or Vismodegib or Cixutumumab or teniposide or Adriamycin or vindesine or nivolumab or ipilimumab or Atezolizumab OR Pembrolizumab OR Avelumab OR Durvalumab

\#5 NSCLC:ti or non-small:ti

\#6 \#1 AND \#2 AND \#3 AND \#4 NOT \#5

Restricted by "Trial" and "2000-"

\section{EMBASE}

('small cell lung cancer':ti OR 'small cell lung carcinoma':ti OR sclc:ti) AND (ed OR extended OR extensive) AND (randomized OR randomised OR rct OR 'phase3' OR 'phase iii' OR randomly OR blinded OR 'blind'/exp OR blind OR 'placebo'/exp OR placebo) AND ('doxorubicin'/ exp OR doxorubicin OR adriamycin OR 'epirubicine'/ exp OR epirubicine OR 'ifosfamide'/exp OR ifosfamide OR 'topotecan'/exp OR topotecan OR 'irinotecan'/exp OR irinotecan OR 'etoposide'/exp OR etoposide OR 'cisplatin'/exp OR cisplatin OR 'gemcitabin'/exp OR gemcitabin OR 'carboplatin'/exp OR carboplatin OR 'epirubicin'/exp OR epirubicin OR 'paclitaxel'/exp OR paclitaxel OR 'apatinib'/exp OR apatinib OR 'amrubicin'/ exp OR amrubicin OR ly2510924 OR 'bevacizumab'/exp OR bevacizumab OR 'vismodegib'/exp OR vismodegib OR 'cixutumumab'/exp OR cixutumumab OR 'teniposide'/ exp OR teniposide OR 'adriamycin'/exp OR adriamycin OR 'vindesine'/exp OR vindesine OR 'nivolumab'/exp OR nivolumab OR 'ipilimumab'/exp OR ipilimumab OR 'atezolizumab'/exp OR atezolizumab OR 'pembrolizumab'/ exp OR pembrolizumab OR 'avelumab'/exp OR avelumab OR 'durvalumab'/exp OR durvalumab) NOT (nsclc:ti OR 
'non small':ti) AND [english]/lim AND ((([article]/lim OR [article in press]/lim OR [conference abstract]/lim OR [conference paper]/lim OR [conference review]/lim OR [letter]/lim) AND [2015-2019]/py) OR ([article]/lim AND [2000-2019]/py))

\section{Supplementary Text 2: Reference list of analyzed trials}

1. Cheng Y, Fan Y, Liu X, et al. Randomized controlled trial of lobaplatin plus etoposide vs. Cisplatin plus etoposide as first-line therapy in patients with extensive-stage small cell lung cancer. Oncol Lett 2019;17:4701-9.

2. Kim DW, Kim HG, Kim JH, et al. Randomized Phase III Trial of Irinotecan Plus Cisplatin versus Etoposide Plus Cisplatin in Chemotherapy-Naïve Korean Patients with Extensive-Disease Small Cell Lung Cancer. Cancer Res Treat 2019;51:119-27.

3. Owonikoko TK, Dahlberg SE, Sica GL, et al. Randomized phase II trial of cisplatin and etoposide in combination with veliparib or placebo for extensive-stage small-cell lung cancer: eCOG-ACRIN 2511 study. J Clin Oncol 2019;37:222-9.

4. Paz-Ares L, Chen Y, Reinmuth N, et al. PL02.11: Overall survival with durvalumab plus platinum-etoposide in firstline extensive-stage SCLC: Results from the CASPIAN study 2019 World Conference on Lung Cancer. Barcelona, Spain 2019. [Conference Abstract].

5. Reck M, Horn L, Novello S, et al. Phase II Study of Roniciclib in Combination with Cisplatin/Etoposide or Carboplatin/Etoposide as First-Line Therapy in Patients with Extensive-Disease Small Cell Lung Cancer. J Thorac Oncol 2019;14:701-11.

6. Weiss JM, Csoszi T, Maglakelidze M, et al. Myelopreservation with the CDK4/6 inhibitor trilaciclib in patients with small cell lung cancer receiving 1st-line chemotherapy: a Phase 1b/randomized Phase 2 trial. Ann Oncol 2019;30:1613-21.

7. Horn L, Mansfield AS, Szczęsna A, et al. First-line atezolizumab plus chemotherapy in extensive-stage smallcell lung cancer. N Engl J Med 2018;379:2220-9.

8. Jalal SI, Lavin P, Lo G, et al. Carboplatin and Etoposide With or Without Palifosfamide in Untreated ExtensiveStage Small-Cell Lung Cancer: A Multicenter, Adaptive, Randomized Phase III Study (MATISSE). J Clin Oncol 2017;35:2619-23.

9. Morikawa N, Inoue A, Sugawara S, et al. Randomized phase II study of carboplatin plus irinotecan versus carboplatin plus amrubicin in patients with chemo-naïve extensive-stage small-cell lung cancer: North Japan Lung Cancer Study Group (NJLCG) 0901. Lung Cancer 2017;111:38-42.

10. Salgia R, Stille JR, Weaver RW, et al. A randomized phase II study of LY2510924 and carboplatin/etoposide versus carboplatin/etoposide in extensive-disease small cell lung cancer. Lung Cancer 2017;105:7-13.

11. Sanborn RE, Patel JD, Masters GA, et al. A Randomized, Double-Blind, Phase 2 Trial of Platinum Therapy Plus Etoposide With or Without Concurrent Vandetanib (ZD6474) in Patients With Previously Untreated Extensive-Stage Small Cell Lung Cancer: Hoosier Cancer Research Network LUN06-113. Cancer 2017;123:303-11.

12. Seckl MJ, Ottensmeier CH, Cullen M, et al. Multicenter, Phase III, Randomized, Double-Blind, Placebo-Controlled Trial of Pravastatin Added to First-Line Standard Chemotherapy in Small-Cell Lung Cancer (LUNGSTAR). J Clin Oncol 2017;35:1506-14.

13. Tiseo M, Boni L, Ambrosio F, et al. Italian, Multicenter, Phase III, Randomized Study of Cisplatin Plus Etoposide With or Without Bevacizumab as First-Line Treatment in Extensive-Disease Small-Cell Lung Cancer: The GOIRCAIFA FARM6PMFJM Trial. J Clin Oncol 2017;35:1281-7.

14. Oh IJ, Kim KS, Park CK, et al. Belotecan/cisplatin versus etoposide/cisplatin in previously untreated patients with extensive-stage small cell lung carcinoma: a multi-center randomized phase III trial. BMC Cancer 2016;16:690.

15. Reck M, Luft A, Szczesna A, et al. Phase III Randomized Trial of Ipilimumab Plus Etoposide and Platinum Versus Placebo Plus Etoposide and Platinum in Extensive-Stage Small-Cell Lung Cancer. J Clin Oncol 2016;34:3740-8.

16. Sun Y, Cheng Y, Hao X, et al. Randomized phase III trial of amrubicin/cisplatin versus etoposide/cisplatin as firstline treatment for extensive small-cell lung cancer. BMC Cancer 2016;16:265.

17. Beniwal SK, Narayan S, Rao GS, et al. Comparision of irinotecan/cisplatin with etoposide/cisplatin in NorthWest Indian population with extensive-stage disease smallcell lung cancer. Ann Oncol 2015;26:ix142.

18. Lu S, Li L, Luo Y, et al. A multicenter, open-label, randomized phase II controlled study of rh-endostatin (Endostar) in combination with chemotherapy in previously untreated extensive-stage small-cell lung cancer. J Thorac Oncol 2015;10:206-211.

19. Shi $\mathrm{Y}, \mathrm{Hu} \mathrm{Y}, \mathrm{Hu} \mathrm{X}$, et al. Cisplatin combined with irinotecan or etoposide for untreated extensive-stage small cell lung cancer: A multicenter randomized controlled 
clinical trial. Thorac Cancer 2015;6:785-91.

20. Langer CJ, Albert I, Ross HJ, et al. Randomized phase II study of carboplatin and etoposide with or without obatoclax mesylate in extensive-stage small cell lung cancer. Lung Cancer 2014;85:420-8.

21. Satouchi M, Kotani Y, Shibata T, et al. Phase III study comparing amrubicin plus cisplatin with irinotecan plus cisplatin in the treatment of extensive-disease small-cell lung cancer: JCOG 0509. J Clin Oncol 2014;32:1262-8.

22. Sekine I, Okamoto H, Horai T, et al. A randomized phase III study of single-agent amrubicin vs. carboplatin/ etoposide in elderly patients with extensive-disease smallcell lung cancer. Clin Lung Cancer 2014;15:96-102.

23. Fink TH, Huber RM, Heigener DF, et al. Topotecan/ cisplatin compared with cisplatin/etoposide as first-line treatment for patients with extensive disease small-cell lung cancer: final results of a randomized phase III trial. J Thorac Oncol 2012;7:1432-9.

24. Schmittel A, Sebastian M, Fischer von Weikersthal L, et al. A German multicenter, randomized phase III trial comparing irinotecan-carboplatin with etoposidecarboplatin as first-line therapy for extensive-disease smallcell lung cancer. Ann Oncol 2011;22:1798-804.

25. Spigel DR, Townley PM, Waterhouse DM, et al. Randomized phase II study of bevacizumab in combination with chemotherapy in previously untreated extensive-stage small-cell lung cancer: results from the SALUTE trial. J Clin Oncol 2011;29:2215-22.

26. Zatloukal P, Cardenal F, Szczesna A, et al. A multicenter international randomized phase III study comparing cisplatin in combination with irinotecan or etoposide in previously untreated small-cell lung cancer patients with extensive disease. Ann Oncol 2010;21:1810-6.

27. Lara Jr PN, Natale R, Crowley J, et al. Phase III trial of irinotecan/cisplatin compared with etoposide/cisplatin in extensive-stage small-cell lung cancer: Clinical and pharmacogenomic results from SWOG S0124. J Clin Oncol 2009;27:2530-5.

28. Lee SM, James LE, Qian W, et al. Comparison of gemcitabine and carboplatin versus cisplatin and etoposide for patients with poor-prognosis small cell lung cancer. Thorax 2009;64:75-80.

29. Socinski MA, Smit EF, Lorigan P, et al. Phase III study of pemetrexed plus carboplatin compared with etoposide plus carboplatin in chemotherapy-naive patients with extensivestage small-cell lung cancer. J Clin Oncol 2009;27:4787-92.

30. Dimitroulis J, Rapti A, Stathopoulos GP, et al. Comparison of cisplatin-paclitaxel combination versus cisplatin- etoposide in patients with small-cell lung cancer: a Phase III study. Oncol Rep 2008;20:879-84.

31. Hermes A, Bergman B, Bremnes R, et al. Irinotecan plus carboplatin versus oral etoposide plus carboplatin in extensive small-cell lung cancer: a randomized phase III trial. J Clin Oncol 2008;26:4261-7.

32. Rudin CM, Salgia R, Wang XF, et al. Randomized phase II study of carboplatin and etoposide with or without the bcl-2 antisense oligonucleotide oblimersen for extensivestage small-cell lung cancer: CALGB 30103. J Clin Oncol 2008;26:870-876.

33. Sekine I, Nokihara H, Takeda K, et al. Randomised phase II trial of irinotecan plus cisplatin vs irinotecan, cisplatin plus etoposide repeated every 3 weeks in patients with extensive-disease small-cell lung cancer. Br J Cancer 2008;98:693-6.

34. Okamoto H, Watanabe K, Kunikane H, et al. Randomised phase III trial of carboplatin plus etoposide vs split doses of cisplatin plus etoposide in elderly or poor-risk patients with extensive disease small-cell lung cancer: JCOG 9702. Br J Cancer 2007;97:162-9.

35. Eckardt JR, von Pawel J, Papai Z, et al. Open-label, multicenter, randomized, phase III study comparing oral topotecan/cisplatin versus etoposide/cisplatin as treatment for chemotherapy-naive patients with extensive-disease small-cell lung cancer. J Clin Oncol 2006;24:2044-51.

36. Hanna N, Bunn PA Jr, Langer C, et al. Randomized phase III trial comparing irinotecan/cisplatin with etoposide/ cisplatin in patients with previously untreated extensivestage disease small-cell lung cancer. J Clin Oncol 2006;24:2038-43.

37. Socinski MA, Weissman C, Hart LL, et al. Randomized phase II trial of pemetrexed combined with either cisplatin or carboplatin in untreated extensive-stage small-cell lung cancer. J Clin Oncol 2006;24:4840-7.

38. Greco FA, Thompson DS, Morrissey LH, et al. Paclitaxel/ carboplatin/etoposide versus paclitaxel/topotecan for extensive-stage small cell lung cancer: A minnie pearl cancer research network randomized, prospective phase II trial. Oncologist 2005;10:728-33.

39. Niell HB, Herndon IJE, Miller AA, et al. Randomized phase III intergroup trial of etoposide and cisplatin with or without paclitaxel and granulocyte colony-stimulating factor in patients with extensive-stage small-cell lung cancer: Cancer and Leukemia Group B trial 9732. J Clin Oncol 2005;23:3752-9.

40. Quoix E, Breton JL, Gervais R, et al. A randomised phase II study of the efficacy and safety of intravenous topotecan 
in combination with either cisplatin or etoposide in patients with untreated extensive disease small-cell lung cancer. Lung Cancer 2005;49:253-61.

41. Lyss AP, Herndon JE 2nd, Lynch TJ Jr, et al. Novel doublets in extensive-stage small-cell lung cancer: a randomized phase II study of topotecan plus cisplatin of paclitaxel (CALGB 9430). Clin Lung Cancer 2002;3:205-10.

42. Noda K, Nishiwaki Y, Kawahara M, et al. Irinotecan plus cisplatin compared with etoposide plus cisplatin for extensive small-cell lung cancer. N Engl J Med 2002;346:85-91. 